\title{
Association of the TNF- $\alpha$ G-308A polymorphism with TNF-inhibitor response in sarcoidosis
}

\author{
Petal A. Wijnen ${ }^{1}$, Johanna P. Cremers ${ }^{2}$, Patty J. Nelemans ${ }^{3}$, Roel J. Erckens ${ }^{4}$, \\ Elske Hoitsma ${ }^{5}$, Tim L. Jansen ${ }^{6}$, Otto Bekers ${ }^{1}$ and Marjolein Drent ${ }^{2,7}$
}

\author{
Affiliations: \\ ${ }^{1}$ Central Diagnostic Laboratory, Maastricht University Medical Centre, Maastricht, The Netherlands. \\ ${ }^{2}$ Dept of Interstitial Lung Diseases, Gelderse Vallei Hospital, Ede, The Netherlands. \\ ${ }^{3}$ Dept of Epidemiology, Maastricht University, Maastricht, The Netherlands. \\ ${ }^{4}$ Dept of Ophthalmology, Maastricht University Medical Centre, Maastricht, The Netherlands. \\ ${ }^{5}$ Dept of Neurology, Diaconessenhuis, Leiden, The Netherlands. \\ ${ }^{6}$ Dept of Rheumatology, University Medical Centre St. Radboud, Nijmegen, The Netherlands. \\ ${ }^{7}$ Dept of Toxicology, Maastricht University, Maastricht, The Netherlands.
}

\section{Correspondence:}

M. Drent, Maastricht University, Faculty of Health, Medicine and Life Science, Dept of Toxicology, PO Box 18, 6720 AA Bennekom, The Netherlands.

E-mail: m.drentamaastrichtuniversity.nl

ABSTRACT Responsiveness to tumour necrosis factor (TNF) inhibitors has been associated with the TNF- $\alpha$ G-308A polymorphism in rheumatoid arthritis. The aim of this study was to examine the association between the presence of this polymorphism and the response to TNF inhibitors in patients with refractory sarcoidosis.

Patients $(\mathrm{n}=111)$ who started TNF-inhibitor treatment (76 infliximab, 35 adalimumab) were followed for at least 1 year. The main symptoms in these patients were fatigue $(n=100,90.1 \%)$, small fibre neuropathy $(n=91,82.0 \%)$, pulmonary involvement $(n=69,62.2 \%)$, and/or uveitis $(n=31,27.9 \%)$. Patients were additionally genotyped for the presence of the TNF- $\alpha$ G-308A polymorphism. Treatment response was assessed using clinical outcome measures and questionnaires.

Three-quarters $(n=83,74.8 \%)$ of the patients responded well. Of the patients without the variant A-allele 93.6\% (73 out of $78, \mathrm{p}<0.001$ ) improved, while $30.3 \%$ (10 out of 33 ) of variant A-allele carriers responded favourably to TNF inhibitors. For patients with the GG-genotype, the probability of improving compared with remaining stable or deteriorating was three times higher (risk ratio 3.09, 95\% CI 1.84-5.20).

Sarcoidosis patients without the TNF- $\alpha-308$ A variant allele (GG-genotype) had a three-fold higher response to TNF inhibitors (adalimumab or infliximab). Further research is needed to evaluate the value of genotyping for the TNF- $\alpha$ G-308A polymorphism in order to tailor TNF-inhibitor treatment.

@ERSpublications

TNF- $\alpha$ G-308A polymorphism predicts TNF-inhibitor response in refractory sarcoidosis: a step to personalised medicine? http://ow.ly/sQ7KO 


\section{Introduction}

Sarcoidosis is a multisystem disease characterised by inflammatory activity with the formation of noncaseating granulomas that commonly affect the lungs, although other organ systems can also be involved $[1,2]$. Clinical presentation and outcome vary greatly and therapeutic options range from no treatment to a variety of pharmacological agents [2].

Although the exact aetiology is still unknown, tumour necrosis factor (TNF)- $\alpha$ is known to play a crucial role in the granuloma formation [2]. The production of this cytokine by alveolar macrophages is substantially elevated and TNF- $\alpha$ elevation has been shown to correlate with sarcoid disease activity and progression [3]. In severe sarcoidosis, the release of inflammatory mediators causes derangement of organ physiology and ultimately irreversible organ damage [1]. Timely implementation of an appropriate potent individual treatment regimen is especially important for patients with a severe disease course and poor prognosis $[4,5]$.

Glucocorticosteroids are traditionally considered first-line treatment, but their long-term use can be impaired by failure or intolerance, with serious adverse effects such as weight gain/obesity, diabetes or osteoporosis $[6,7]$. In case of intolerable side-effects or steroid resistance, second-line treatment options should be considered, such as methotrexate (MTX), azathioprine or leflunomide, but these therapies may also be subject to failure or intolerance [8-10]. Another option to consider is TNF inhibitors, which inhibit the potent pro-inflammatory TNF- $\alpha$, often a predominant problem in patients with refractory sarcoidosis, and these have shown promising results [11-14]. However, a drawback of TNF inhibitors is considerable treatment costs [15]. Therefore, the ideal situation would involve identification of patients likely to respond to TNF inhibitors before starting treatment.

When considering the natural course of sarcoidosis, polymorphisms seem to make an important contribution to the clinical phenotype [16]. Genetic analysis has previously revealed a number of polymorphisms in genes coding for TNF- $\alpha$, with potential functional consequences [17]. The presence of the TNF- $\alpha-308 \mathrm{~A}$ variant allele, which has been associated with variations in TNF- $\alpha$ production, proved to be associated with a favourable prognosis [16-18]. In rheumatoid arthritis, the TNF- $\alpha$ G-308A polymorphism has been studied in relation to TNF inhibitors. Patients without the TNF- $\alpha-308 \mathrm{~A}$ variant allele were found to be better responders $[19,20]$. However, studies assessing such an association in sarcoidosis are currently lacking.

The aim of the present study was to assess the association between the presence of the TNF- $\alpha$ G-308A polymorphism and the response to TNF inhibitors among patients with refractory sarcoidosis.

\section{Methods}

\section{Patients}

Dutch Caucasian patients with refractory sarcoidosis $(n=118)$ with various manifestations despite conventional treatment were started on TNF inhibitors during a 4-year period (2007-2011) and followed for at least 1 year. Patients were diagnosed with sarcoidosis in accordance with the guidelines of the World Association of Sarcoidosis and Other Granulomatous diseases, as previously reported $[1,18]$.

All patients were given prednisone as a first-line treatment. When this treatment failed or when serious sideeffects occurred, a combination of prednisone and MTX was given. Finally, TNF-inhibitor treatment was started if combination treatment did not achieve clinical improvement. Failure of therapy was determined by a pulmonologist, neurologist and/or ophthalmologist, and defined as either clinical or symptomatic progression of disease despite conventional therapy.

If treatment was discontinued within 1 year, patients were regarded as drop-outs. Of the initial 118 patients, seven discontinued treatment within 1 year, leaving 111 for the analyses. Pulmonary and extrapulmonary (i.e. ocular, cutaneous, lymphatic, musculoskeletal and neurological) data were collected. Patients were treated with infliximab $(n=76)$ or adalimumab $(n=35)$.

The study was performed in accordance with the Declaration of Helsinki and its amendments. Written informed consent for participation was obtained from all patients and the protocol was approved by the Medical Ethics Board of Maastricht University Medical Centre, Maastricht, the Netherlands (METC 11-4-116).

\section{Clinical response rate}

Responses to TNF inhibitors were defined. A patient's individual clinical response was graded 1 year after starting TNF inhibitors, based on documented objective data. Each organ with documented sarcoid involvement or symptom or inflammation level was graded in a systematic manner before and after 1 year of TNF-inhibitor treatment, as described below. We carefully documented whether a patient improved, was stable or deteriorated. 
Improvement was defined as an increase or decrease in at least one of the parameters, values or scores by $\geqslant 10 \%$ of the original value. Any change, whether an increase or a reduction, of $<10 \%$ was regarded as stable disease and a decrease or increase, when appropriate, of $\geqslant 10 \%$ as deterioration.

\section{Evaluation of pulmonary involvement}

All chest radiographs were graded, at inclusion and after 1 year of treatment, by a single observer, who was blinded to the genetic test results. Radiographic abnormalities were classified into five stages ( 0 to IV), as described previously [18].

Forced vital capacity (FVC), forced expiratory volume in $1 \mathrm{~s}$ (FEV1) and diffusing capacity of the lung for carbon monoxide (DLCO) were measured [18]. Values were expressed as a percentage of the predicted values. The cut-off value for DLCO, FEV1 and FVC was $<80 \%$ predicted ( $\geqslant 80 \%$ was considered normal) [21]. Respiratory functional impairment (RFI) was defined as DLCO $<80 \%$ pred, FVC $<80 \%$ pred or FEV1 $<80 \%$ pred. Patients without RFI were those for whom all three indices were normal [22]. Patients with pulmonary sarcoidosis should have RFI and/or abnormal chest radiographs (stage II or higher) (fig. 1).

\section{Evaluation of uveitis}

All patients with eye problems due to sarcoidosis were evaluated by the same ophthalmologist, who was blinded to the genetic test results. The patients were screened for the presence of uveitis, as described previously [23]. In addition, fluorescein angiography or optical coherence tomography was performed to detect the presence of vasculitis, papillitis or macular oedema, as described previously (fig. 2) [23].

The overall outcome of intraocular inflammatory signs was scored as improvement if at least one of the inflammatory signs scored showed complete clearance or if at least one of the inflammatory signs scored improved in the absence of deterioration of other inflammatory signs. It was scored as stabilisation if all inflammatory signs remained unchanged and as deterioration if at least one of the inflammatory signs increased [23].

\section{Evaluation of small fibre neuropathy}

The presence of small fibre neuropathy (SFN) symptoms was assessed using the Small Fiber Neuropathy Screenings List (SFNSL). It is a practical, brief and easy to use tool to screen for the presence of SFN-related symptoms with high scores indicating more severe SFN-related symptoms [24].

Temperature threshold testing was used to support the presence of SFN. This test was used to assess the function of small calibre sensory fibres by measuring temperature sensation thresholds, as described previously [25].

\section{Evaluation of fatigue}

One of the most important patient-reported outcome measures is fatigue, which was assessed by means of the Fatigue Assessment Scale (FAS). The FAS contains 10 specific fatigue questions that have been validated for sarcoidosis patients, with high scores indicating more fatigue. The minimal clinically important difference has been established, and a change of $\geqslant 4$ points or $10 \%$ change in FAS score was found to be clinically significant [26].

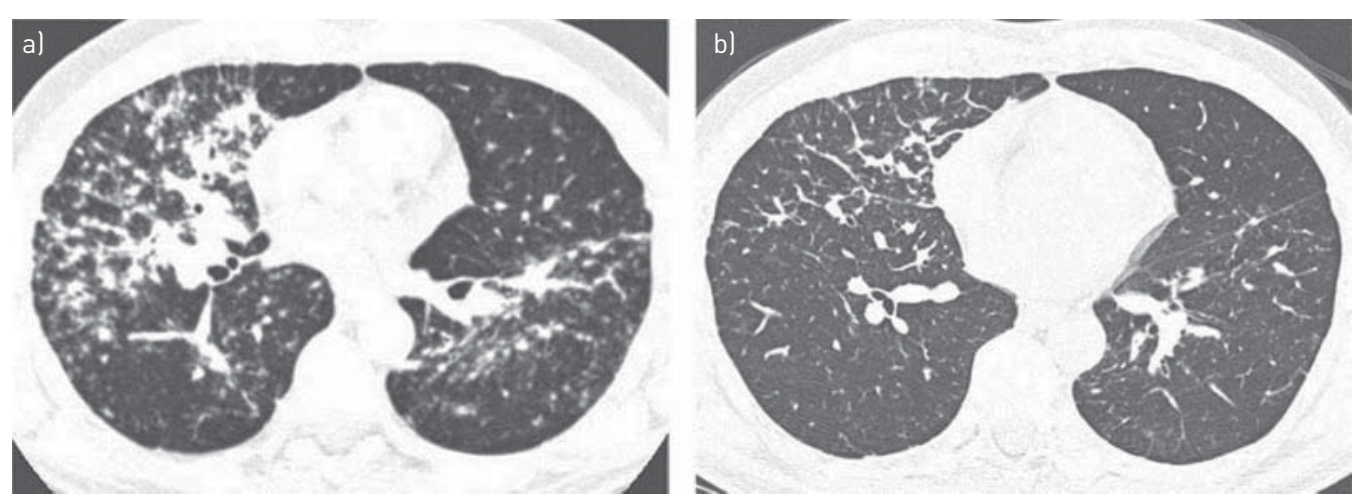

FIGURE 1 An example of a patient with respiratory functional impairment with substantial improvement after 1 year of treatment with infliximab. The original value of the diffusing capacity of the lung for carbon monoxide improved by $\geqslant 10 \%$ from $67 \%$ predicted to $78 \%$ pred, while the forced vital capacity changed from $77 \%$ pred to $83 \%$ pred and the forced expiratory volume in $1 \mathrm{~s}$ from $90 \%$ pred to $92 \%$ pred. The high resolution computed tomography scan a) before and b) after treatment with infliximab showed a decrease in the parenchymal lesions. 

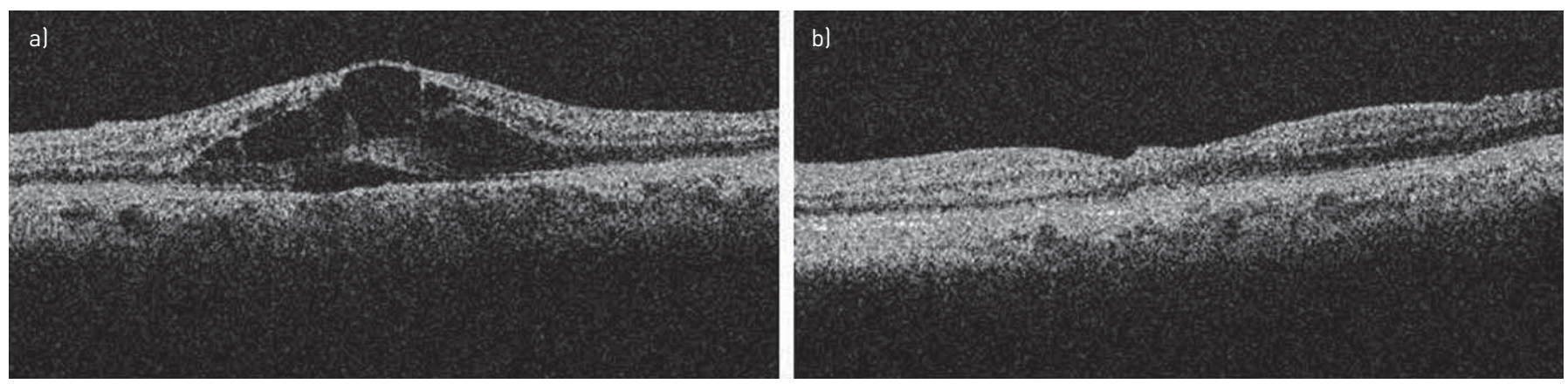

FIGURE 2 An example of an optical coherence tomography scan in a patient with uveitis a) before and b) after 1 year of treatment with adalimumab, showing reduction of macular oedema.

\section{Evaluation of inflammation}

Serum levels of angiotensin-converting enzyme (reference interval 9-25 units $\cdot \mathrm{L}^{-1}$ ) were measured using a colorimetric method (FU 116; Fujirebio Inc., Tokyo, Japan) and soluble interleukin-2 receptor (levels considered elevated if $>3154 \mathrm{pg} \cdot \mathrm{mL}^{-1}$ ) were measured using commercially available Diaclone ELISA kits (Sanquin, Amsterdam, the Netherlands) according to the manufacturer's instructions.

\section{Other manifestations}

Neurosarcoidosis was evaluated by a neurologist based on the results of magnetic resonance imaging scans (fig. 3). Photographs of skin lesions prior to and after treatment were used as the main outcome measures for skin lesions (fig. 4).

\section{Evaluation of response rates}

The overall response rate was calculated by scoring improvement, stable disease or deterioration of disease manifestations of sarcoidosis. Response was defined as improvement in at least one of the following if it was the main reason for treatment, RFI, SFN symptoms, uveitis, skin lesions or neurosarcoidosis, without deterioration of any other disease manifestation. Stable was defined as no improvement or no deterioration
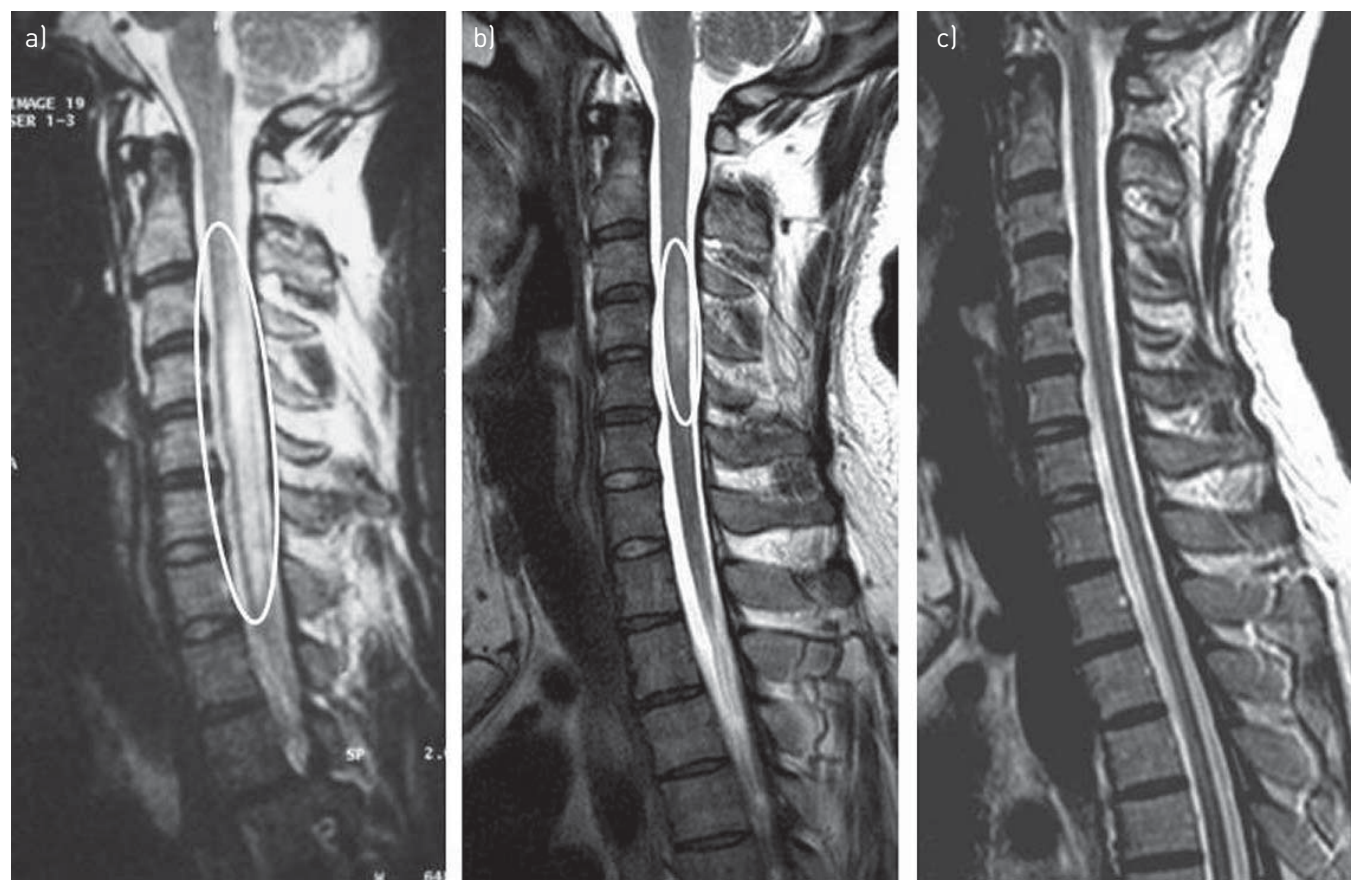

FIGURE 3 Sagittal T2 weighted magnetic resonance images. a) Before treatment showing an extensive spinal cord lesion. b) After 1 year of treatment with infliximab, with only a minor lesion left. c) After 3 years, showing complete remission of the spinal cord lesion. 
of at least the major reason for treatment without deterioration of any other disease manifestation. Deterioration was defined as deterioration of at least the major disease manifestation.

\section{Genotyping}

DNA was obtained using venous EDTA anticoagulated blood and isolated, according to the manufacturer's instructions. All patients were genotyped for the TNF- $\alpha$ G-308A promoter polymorphism using a real-time PCR fluorescence resonance energy transfer assay (TIB MOLBIOL, Berlin, Germany), as described previously [18]. The person who performed the analyses was blinded to the clinical data and response to therapy.

\section{Statistical analysis}

Statistical analyses were performed with SPSS 20.0 (SPSS Inc., Chicago, IL, USA).

Cross tables were used to compare the percentages of patients with response according to each genotype. Relative risk (95\% CI) was calculated to evaluate strength of the association. Multivariate logistic regression analyses were used to adjust for relevant baseline differences between genotype groups. The Chi-squared test was used to test for statistically significant differences between groups and to calculate deviations from the Hardy-Weinberg equilibrium. $\mathrm{p}<0.05$ (two-sided) was considered to indicate statistical significance.

\section{Results}

Characteristics of patients in our sarcoidosis sample and the main reasons for initiating TNF inhibitors are summarised in table 1.

Patients suffered from one or more disabling problem: 91 (82.0\%) out of 111 suffered from SFN (SFNSL $>11$ ), with $38(34.2 \%)$ having severe symptoms (SFNSL >37); $69(62.2 \%)$ out of 111 suffered from pulmonary sarcoidosis (fig. 1); 31 (27.9\%) out of 111 from uveitis; six (5.4\%) out of 111 from skin lesions; one $(0.9 \%)$ out of 111 from spinal cord involvement; and one $(0.9 \%)$ out of 111 from kidney failure. In addition, the majority of the patients $(100(90.1 \%)$ out of 111$)$ also suffered from fatigue (FAS $\geqslant 22)$, with $59(59.0 \%)$ out of 100 having severe fatigue (FAS $>34$ ).

Responders were identified based on the following criteria. In 39 (56.5\%) out of the 69 patients with RFI at least one lung function test parameter improved by $>10 \%$. Of the 31 patients with eye involvement, 23 (74.2\%) improved substantially according to the criteria used by the ophthalmologist (fig. 2). Neurological symptoms improved in $49(53.8 \%)$ out of 91 patients, in 17 of whom this appeared to be the only improving primary end-point. All six patients with skin lesions responded (fig. 4), as did the patient suffering from neurosarcoidosis (spinal cord involvement) (fig. 3) and the patient with kidney problems. Signs of activity and inflammation improved in 75 (67.6\%) out of 111 patients, and fatigue improved in 60 $(60.0 \%)$ out of 100 patients. None of the responders showed deterioration in any of the other manifestations of sarcoidosis that were present.

Overall, $83(74.8 \%)$ of the 111 included patients with refractory sarcoidosis had a positive response. Improvement was achieved in $119(59.8 \%)$ of the 199 organs assessed. The choice of TNF inhibitor had no
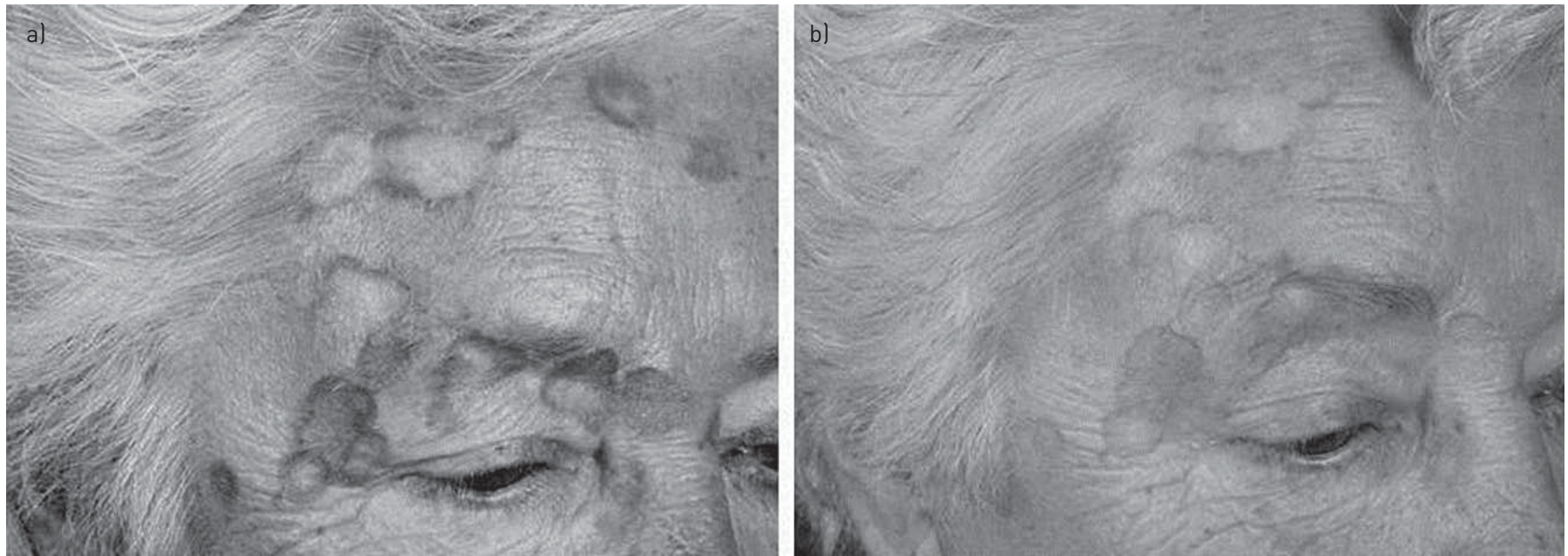

FIGURE 4 An example of a patient with skin manifestations. Photographs taken a) before and b) after 1 year of treatment with infliximab, showing a reduction of the intensity of the facial skin lesions. 


\begin{tabular}{|c|c|c|c|c|}
\hline & Total population & Lung involvement & SFN symptoms & Eye involvement \\
\hline Subjects & 111 & 69 & 32 & 10 \\
\hline Age years & $45.0 \pm 10.6$ & $44.5 \pm 10.8$ & $46.6 \pm 9.8$ & $43.0 \pm 11.6$ \\
\hline CXR stage $0+\mathrm{I} / \mathrm{II}+\mathrm{III} / \mathrm{IV}$ & $54 / 43 / 14$ & $21 / 34 / 14^{* * *}$ & $24 / 8 / 0$ & $9 / 1 / 0$ \\
\hline FEV1 $\%$ predicted & $85.7 \pm 24.2$ & $75.2 \pm 22.7 * * *$ & $104.5 \pm 12.1$ & $105.4 \pm 13.4$ \\
\hline FVC $\%$ predicted & $96.6 \pm 19.2$ & $88.6 \pm 17.7^{* * *}$ & $110.6 \pm 13.1$ & $111.7 \pm 12.9$ \\
\hline ACE units $\cdot \mathrm{L}^{-1}$ & $20.7 \pm 12.9$ & $21.5 \pm 14.2$ & $19.0 \pm 10.1$ & $20.0 \pm 9.6$ \\
\hline $\mathrm{sIL-2R} \mathrm{pg} \cdot \mathrm{mL}^{-1}$ & $3073 \pm 3120$ & $3530 \pm 3645$ & $2337 \pm 1460$ & $1719 \pm 1190$ \\
\hline FAS & $33.0 \pm 8.5$ & $33.5 \pm 8.4$ & $33.6 \pm 17.9$ & $27.6 \pm 8.1$ \\
\hline Fatigue reported & $100(90.1)$ & 63 (91.3) & $29(90.6)$ & $8(80.0)$ \\
\hline SFNSL & $32.5 \pm 19.3$ & $32.0 \pm 20.0$ & $37.6 \pm 18.0$ & $18.8 \pm 9.1^{* *}$ \\
\hline SFN symptoms & $91(\overline{8} 2.0)$ & $54(\overline{7} 8.3)$ & $32(\overline{100})$ & $5(50.0)$ \\
\hline Infliximab & 76 & 46 & 27 & 3 \\
\hline mg per dose i.v. & $404.1 \pm 25.5$ & $404.5 \pm 28.1$ & $403.7 \pm 19.2$ & $400.0 \pm 0.0$ \\
\hline Dose interval weeks & $4.5 \pm 0.6$ & $4.5 \pm 0.5$ & $4.6 \pm 0.6$ & $4.0 \pm 0$ \\
\hline \multicolumn{5}{|l|}{ Co-medication } \\
\hline No prednisone, no MTX & 40 & 15 & 16 & 9 \\
\hline Prednisone alone & 14 & 10 & 1 & 3 \\
\hline mg per day \pm range p.o. & $14.3 \pm 10.7$ & $15.4 \pm 11.7$ & $15.0 \pm 0.0$ & $8.8 \pm 8.8$ \\
\hline Prednisone+MTX & 28 & 19 & 7 & 2 \\
\hline prednisone mg per day \pm range p.o. & $9.3 \pm 5.2$ & $8.6 \pm 3.7$ & $12.9 \pm 8.1$ & $5.0 \pm 0.0$ \\
\hline MTX mg per week \pm range p.o. & $9.1 \pm 2.9$ & $9.6 \pm 3.1$ & $7.9 \pm 1.0$ & $8.5 \pm 5.3$ \\
\hline MTX alone & 29 & $\frac{1}{18}$ & $\frac{1}{8}$ & $\frac{1}{3}$ \\
\hline mg per week \pm range p.o. & $7.5 \pm 1.4$ & $7.5 \pm 0.8$ & $7.5 \pm 5.5$ & $7.5 \pm 0.0$ \\
\hline
\end{tabular}

Data are presented as $n$, mean \pm SD or $n(\%)$, unless otherwise stated. SFN: small fibre neuropathy; CXR: chest radiography; FEV1: forced expiratory volume in $1 \mathrm{~s}$; FVC: forced vital capacity; DLCO: diffusing capacity of the lung for carbon monoxide; RFI: respiratory functional impairment defined as DLCO $<80 \%$ predicted, FVC $<80 \%$ pred or FEV1 $<80 \%$ pred; ACE: angiotensin-converting enzyme (reference range $9.0-25.0$ units. $\mathrm{L}^{-1}$ ); sIL-2R: soluble interleukin-2 receptor (reference range $<3154 \mathrm{pg} \cdot \mathrm{mL}^{-1}$ ); FAS: fatigue assessment scale; SFNSL: Small Fiber Neuropathy Screenings List; MTX: methotrexate. ${ }^{* *}: \mathrm{p}<0.01$ for eye involvement versus lung involvement and SFN; ${ }^{* *}: \mathrm{p}<0.001$ for lung involvement versus SFN and eye involvement.

influence on outcome, as both infliximab and adalimumab resulted in 25\% nonresponders. Allele frequencies were $84.2 \%$ for the G-allele and $15.8 \%$ for the A-allele. The frequency distribution is in agreement with the Hardy-Weinberg equilibrium. There were no significant differences in TNF- $\alpha$ G-308A polymorphism distribution between the various disease manifestations.

Of the patients without the A-allele, $73(93.6 \%)$ out of $78(\mathrm{p}<0.001)$ had a positive response to TNF inhibitors, compared with $10(30.3 \%)$ out of the 33 patients with the variant A-allele. For patients possessing the GG-genotype, the probability of improving was three times higher than that of remaining stable or deteriorating (relative risk 3.09, 95\% CI 1.84-5.20) (table 2).

The different genotype groups (GG 70.3\%, GA 27.9\%, and AA 1.8\%) were similar with respect to distribution of therapy (infliximab versus adalimumab) and co-medication, but the group with the GG-genotype had a higher mean age and proportion of female patients (table 3). Multivariate logistic regression analysis to adjust for baseline differences in age and sex between the genotype groups resulted in an adjusted odds ratio of 39.7 (95\% CI 11.1-142.3), which was slightly higher than the unadjusted odds ratio of 33.6 (95\% CI 10.4-108.3).

Nine $(8.1 \%)$ patients formed antibodies against infliximab and were switched to adalimumab $(\mathrm{n}=4)$ or had to discontinue the TNF-inhibitor treatment $(n=5)$. None of the patients developed antibodies against adalimumab. Adverse events were seen in 14 (12.6\%) out of 111 patients treated with TNF inhibitors. Documented adverse events were minor infections $(n=11)$, sepsis $(n=1)$ and the occurrence of herpes 
TABLE 2 Disease course in relation to tumour necrosis factor- $\alpha$ G-308A polymorphism

\begin{tabular}{lccccc} 
Genotype & Total & Improved & $\begin{array}{c}\text { Stabilised/ } \\
\text { worsened }\end{array}$ & $\begin{array}{c}\text { Relative risk } \\
(95 \% \text { CI) }\end{array}$ & p-value \\
\hline GG & 78 & $73(93.6)$ & $5(6.4)$ & $3.09(1.84-5.20)$ & $<0.001$ \\
GA & 31 & $9(29.0)$ & $22(71.0)$ & 1 & \\
AA & 2 & $1(50.0)$ & $1(50.0)$ & & \\
\hline
\end{tabular}

Date are presented as $\mathrm{n}$ or $\mathrm{n}(\%)$, unless otherwise stated. GG: wild type variant; GA: heterozygote variant; AA: homozygote variant.

zoster infection $(n=5)$. Two patients suffered from an infusion reaction, after the fifth and eighth infliximab administrations, respectively. However, after adjustment of the infusion time no further problems emerged and the treatment was continued.

\section{Discussion}

To the best of our knowledge, this is the first study to demonstrate an association between the TNF- $\alpha$ G-308A polymorphism and responsiveness to TNF-inhibitor treatment in sarcoidosis. Efficacy of TNF inhibitors was found in $75 \%$ of our sample of patients with refractory sarcoidosis who had failed to respond to conventional treatment. TNF-inhibitor treatment was well tolerated. Patients without the TNF- $\alpha-308 \mathrm{~A}$ variant allele (GG-genotype) responded better to TNF inhibitors (93.6\% versus only $30.3 \%$ ), having a threefold higher probability of improving compared with variant allele carriers (relative risk 3.09, 95\% CI 1.84-5.20). This suggests that this variant allele (GA/AA genotypes) negatively affects TNF-inhibitor treatment response. However, since the study population was relatively small, the associations should be interpreted with some caution.

It is well known that the clinical course and prognosis of sarcoidosis varies considerably, and research suggests that polymorphisms coding for TNF- $\alpha$ production might play a role $[16,17]$. The TNF- $\alpha-308 \mathrm{~A}$ variant allele is associated with higher TNF- $\alpha$ production, and several studies have found the variant allele to be more frequently observed in patients with Löfgren's syndrome, who often showed spontaneous remission $[16,17,27]$. The presence of the TNF- $\alpha-308 \mathrm{~A}$ variant allele has been found to have a more favourable impact on clinical outcome [18]. By contrast, the GG-genotype has been associated with an unfavourable prognosis, with a significantly higher risk of developing persistent sarcoidosis and progression to more severe pulmonary involvement [18].

TABLE 3 Demographic and medication characteristics of the sarcoidosis sample according to tumour necrosis factor- $\alpha$ G-308A polymorphism

\begin{tabular}{|c|c|}
\hline \multicolumn{2}{|c|}{ Genotype } \\
\hline GG & GA/AA \\
\hline
\end{tabular}

\begin{tabular}{|c|c|c|c|}
\hline Subjects $n$ & 78 & 33 & \\
\hline \multicolumn{4}{|l|}{ Sex } \\
\hline Female & 35 (44.9) & 11 (33.3) & 0.30 \\
\hline Male & $43(55.1)$ & $22(66.7)$ & \\
\hline Age at diagnosis years & $45.7 \pm 10.3$ & $42.6 \pm 10.8$ & 0.15 \\
\hline \multicolumn{4}{|l|}{ Therapy } \\
\hline Infliximab" & $52(66.7)$ & $24(72.7)$ & 0.66 \\
\hline Adalimumab & 26 (33.3) & $9(27.3)$ & \\
\hline \multicolumn{4}{|l|}{ Co-medication } \\
\hline None & $27(34.6)$ & 13 (39.5) & 0.97 \\
\hline Prednisone & $10(12.8)$ & $4(12.1)$ & \\
\hline Prednisone+MTX & $20(25.6)$ & $8(24.2)$ & \\
\hline MTX & $21(27.0)$ & $8(24.2)$ & \\
\hline
\end{tabular}

Data are presented as $\mathrm{n}(\%)$ or mean $\pm \mathrm{SD}$, unless otherwise stated. $\mathrm{GG}$ : wild type variant; $\mathrm{GA}$ : heterozygote variant; AA: homozygote variant; MTX: methotrexate. ${ }^{\#}: \mathrm{n}=76 ;{ }^{\top}: \mathrm{n}=35$. 
In the present study, sarcoidosis patients with the TNF- $\alpha$ G-308A GG-genotype appeared to be more likely to be TNF-inhibitor responders. The GA- or AA-genotype was found in $29.7 \%$ of our 111 patients, whereas in other studies these genotypes were found in about $35-40 \%$ of the general sarcoidosis populations and/or healthy controls $[17,18,27]$. The slightly lower frequency of the AA- and GA-genotypes in our study population could be the result of selection bias, as more severe sarcoidosis cases tend to undergo TNFinhibitor therapy. The GG-genotype is more likely to be present in these cases, since this genotype has been associated with a more unfavourable sarcoidosis prognosis [18].

The association between the TNF- $\alpha$ G-308A polymorphism and TNF-inhibitor therapy has been more extensively studied in rheumatoid arthritis. In line with our results, rheumatoid arthritis patients with the GG-genotype had a better overall response rate $[19,20,28]$. There have been no previous studies in sarcoidosis demonstrating this association. It was previously shown that patients with a severe disease course were more likely to respond to TNF-inhibitor treatment [11,29]. It is tempting to speculate that these patients most likely did not possess the variant A-allele. Since GG-genotype carriers are more likely to have an unfavourable outcome and to achieve a good response to TNF-inhibitor treatment, the decision whether or not to start TNF inhibitors in these patients seems rather easy. In contrast, patients suffering from sarcoidosis with the variant A-allele are more likely to have a favourable prognosis with spontaneous resolution of the disease. Nevertheless, in the present study approximately 70\% (23 out of 33) of these patients did not respond to TNF inhibitors. A considerable proportion of the variant allele carriers showed stabilisation of disease. Although stabilisation was not considered a "response" in the present study, in specific cases it might be regarded as a desirable aim. To date, too little evidence is available to decide whether these patients should not be given TNF-inhibitor treatment.

The results of our study are in line with other case series demonstrating efficacy of infliximab in $64-100 \%$ of sarcoidosis patients treated $[12,30,31]$. In the present study, uveitis demonstrated the best response rate (74\%), followed by RFI (57\%) and SFN (54\%). These response rates are in line with previous research $[11,23,29,31,32]$. It is probable that the higher response rate for uveitis is influenced by the shorter time between diagnosis and initiating TNF inhibitors $(4.5 \pm 4.3$ years for uveitis, compared with $6.3 \pm 5.6$ years for SFN and $6.8 \pm 5.6$ years for RFI; $p=0.05$ ). These results suggest that initiating TNF inhibitors early in the course of the disease might prevent serious organ damage and result in a more favourable outcome. This is in agreement with results in Crohn's disease $[33,34]$.

The TNF inhibitors adalimumab and infliximab were both generally well tolerated in our study, in agreement with data from other studies $[35,36]$. Adverse events were observed in $12.6 \%$ of patients and a $4.5 \%$ discontinuation rate due to antibody formation was seen during at least 1 year of TNF-inhibitor treatment. Outcomes or adverse effects did not differ between infliximab and adalimumab, similar to findings for other chronic inflammatory diseases [34, 37, 38].

Refractory uveitis is a registered indication for adalimumab treatment in the Netherlands, whereas patients with pulmonary and/or neurological symptoms have mainly been treated with infliximab. The choice between adalimumab and infliximab was, therefore, mainly based on insurance reimbursement issues.

Despite the overall efficacy of TNF inhibitors, there are limitations to the use of TNF-inhibitor treatment for every sarcoidosis patient. In addition to the side-effects, another drawback of TNF inhibitors is the considerable treatment cost [15]. It is, therefore, important to tailor treatment. The present study provides a step forward in identifying sarcoidosis patients who might be at risk of developing a more severe manifestation of the disease and in selecting those most likely to have a good response to TNF-inhibitor treatment. It might help to start an appropriate individual treatment regimen and, thus, avoid irreversible damage at an early stage [7]. It is useful to know beforehand whether a patient is highly likely to respond to TNF-inhibitor treatment, as this can be of great clinical and economic benefit. A better understanding may help achieve personalised, cost-effective treatment regimens. However, to date, too little evidence is available to decide whether TNF inhibitors should be withheld from sarcoidosis patients carrying the variant A-allele. For these patients there may be better alternative options. Prospective randomised controlled trials, with a sufficiently large study population, are needed to address the effect of TNF inhibitors in patients with the variant A-allele, compared with placebo and/or conventional therapy.

In conclusion, our results show that sarcoidosis patients without the TNF- $\alpha-308 \mathrm{~A}$ variant allele (GGgenotype) had a three-fold higher response to TNF-inhibitor treatment than A-allele carriers. We also found that TNF inhibitors were efficacious in the treatment of refractory sarcoidosis (75\% response rate) and were well tolerated. Further research is needed to evaluate whether individualised treatment can be improved by basing decisions on the TNF- $\alpha$ G-308A polymorphism of sarcoidosis patients in whom conventional therapy has failed or proved intolerable. 


\section{References}

Statement on sarcoidosis. Joint Statement of the American Thoracic Society (ATS), the European Respiratory Society (ERS) and the World Association of Sarcoidosis and Other Granulomatous Disorders (WASOG) adopted by the ATS Board of Directors and by the ERS Executive Committee, February 1999. Am J Respir Crit Care Med 1999; 160: 736-755.

2 Iannuzzi MC, Fontana JR. Sarcoidosis: clinical presentation, immunopathogenesis, and therapeutics. JAMA 2011; 305: 391-399.

3 Fehrenbach H, Zissel G, Goldmann T, et al. Alveolar macrophages are the main source for tumour necrosis factor- $\alpha$ in patients with sarcoidosis. Eur Respir J 2003; 21: 421-428.

Baughman RP, Costabel U, du Bois RM. Treatment of sarcoidosis. Clin Chest Med 2008; 29: 533-548.

Baughman RP, Nunes H, Sweiss NJ, et al. Established and experimental medical therapy of pulmonary sarcoidosis. Eur Respir I 2013; 41: 1424-1438.

Grutters JC, van den Bosch JM. Corticosteroid treatment in sarcoidosis. Eur Respir J 2006; 28: 627-636.

Baughman RP. Pulmonary sarcoidosis. Clin Chest Med 2004; 25: 521-530.

Paramothayan S, Lasserson T. Treatments for pulmonary sarcoidosis. Respir Med 2008; 102: 1-9.

Sahoo DH, Bandyopadhyay D, Xu M, et al. Effectiveness and safety of leflunomide for pulmonary and extrapulmonary sarcoidosis. Eur Respir J 2011; 38: 1145-1150.

10 Cremers JP, Drent M, Bast A, et al. Multinational evidence-based World Association of Sarcoidosis and Other Granulomatous Disorders recommendations for the use of methotrexate in sarcoidosis: integrating systematic literature research and expert opinion of sarcoidologists worldwide. Curr Opin Pulm Med 2013; 19: 545-561.

11 Baughman RP, Drent M, Kavuru M, et al. Infliximab therapy in patients with chronic sarcoidosis and pulmonary involvement. Am J Respir Crit Care Med 2006; 174: 795-802.

12 Judson MA, Baughman RP, Costabel U, et al. Efficacy of infliximab in extrapulmonary sarcoidosis: results from a randomised trial. Eur Respir J 2008; 31: 1189-1196.

13 Russell E, Luk F, Manocha S, et al. Long term follow-up of infliximab efficacy in pulmonary and extra-pulmonary sarcoidosis refractory to conventional therapy. Semin Arthritis Rheum 2013; 43: 119-124.

14 Vorselaars ADM, Verwoerd A, van Moorsel CHM, et al. Prediction of relapse after discontinuation of infliximab therapy in severe sarcoidosis. Eur Respir J 2014; 43: 602-609.

15 Liu Y, Wu EQ, Bensimon AG, et al. Cost per responder associated with biologic therapies for Crohn's disease, psoriasis, and rheumatoid arthritis. Adv Ther 2012; 29: 620-634.

16 Swider C, Schnittger L, Bogunia-Kubik K, et al. TNF-alpha and HLA-DR genotyping as potential prognostic markers in pulmonary sarcoidosis. Eur Cytokine Netw 1999; 10: 143-146.

17 Seitzer U, Swider C, Stüber F, et al. Tumour necrosis factor alpha promoter gene polymorphism in sarcoidosis. Cytokine 1997; 9: 787-790.

18 Wijnen PA, Nelemans PJ, Verschakelen JA, et al. The role of tumor necrosis factor alpha G-308A polymorphisms in the course of pulmonary sarcoidosis. Tissue Antigens 2010; 75: 262-268.

19 Mugnier B, Balandraud N, Darque A, et al. Polymorphism at position -308 of the tumor necrosis factor alpha gene influences outcome of infliximab therapy in rheumatoid arthritis. Arthritis Rheum 2003; 48: 1849-1852.

20 Zeng Z, Duan Z, Zhang T, et al. Association between tumor necrosis factor-alpha (TNF- $\alpha$ ) promoter -308 G/A and response to TNF- $\alpha$ blockers in rheumatoid arthritis: a meta-analysis. Mod Rheumatol 2013; 23: 489-495.

21 Quanjer PH, Tammeling GJ, Cotes JE, et al. Lung volumes and forced ventilatory flows. Report Working Party Standardization of Lung Function Tests, European Community for Steel and Coal. Official Statement of the European Respiratory Society. Eur Respir J 1993; 6: Suppl. 16, 5-40.

22 Rothkrantz-Kos S, van Dieijen-Visser MP, Mulder PG, et al. Potential usefulness of inflammatory markers to monitor respiratory functional impairment in sarcoidosis. Clin Chem 2003; 49: 1510-1517.

23 Erckens RJ, Mostard RL, Wijnen PA, et al. Adalimumab successful in sarcoidosis patients with refractory chronic non-infectious uveitis. Graefes Arch Clin Exp Ophthalmol 2012; 250: 713-720.

24 Hoitsma E, De Vries J, Drent M. The small fiber neuropathy screening list: construction and cross-validation in sarcoidosis. Respir Med 2011; 105: 95-100.

25 Hoitsma E, De Vries J, van Santen-Hoeufft M, et al. Impact of pain in a Dutch sarcoidosis patient population. Sarcoidosis Vasc Diffuse Lung Dis 2003; 20: 33-39.

26 de Kleijn WP, De Vries J, Wijnen PA, et al. Minimal (clinically) important differences for the Fatigue Assessment Scale in sarcoidosis. Respir Med 2011; 105: 1388-1395.

27 Grutters JC, Sato H, Pantelidis P, et al. Increased frequency of the uncommon tumor necrosis factor -857T allele in British and Dutch patients with sarcoidosis. Am J Respir Crit Care Med 2002; 165: 1119-1124.

28 Marotte H, Miossec P. Biomarkers for prediction of TNF $\alpha$ blockers response in rheumatoid arthritis. Joint Bone Spine 2010; 77: 297-305.

29 Rossman MD, Newman LS, Baughman RP, et al. A double-blinded, randomized, placebo-controlled trial of infliximab in subjects with active pulmonary sarcoidosis. Sarcoidosis Vasc Diffuse Lung Dis 2006; 23: 201-208.

30 Croft AP, Situnayake D, Khair O, et al. Refractory multisystem sarcoidosis responding to infliximab therapy. Clin Rheumatol 2012; 31: 1013-1018.

31 Ulbricht KU, Stoll M, Bierwirth J, et al. Successful tumor necrosis factor alpha blockade treatment in therapyresistant sarcoidosis. Arthritis Rheum 2003; 48: 3542-3543.

32 Parambil JG, Tavee JO, Zhou L, et al. Efficacy of intravenous immunoglobulin for small fiber neuropathy associated with sarcoidosis. Respir Med 2011; 105: 101-105.

33 Beaugerie L, Sokol H. Clinical, serological and genetic predictors of inflammatory bowel disease course. World J Gastroenterol 2012; 18: 3806-3813.

34 D'Haens GR, Panaccione R, Higgins PD, et al. The London position statement of the world congress of gastroenterology on biological therapy for IBD with the European Crohn's and colitis organization: when to start, when to stop, which drug to choose, and how to predict response? Am J Gastroenterol 2011; 106: 199-212.

35 Winthrop KL, Baddley JW, Chen L, et al. Association between the initiation of anti-tumor necrosis factor therapy and the risk of herpes zoster. JAMA 2013; 309: 887-895. 
Grijalva CG, Chen L, Delzell E, et al. Initiation of tumor necrosis factor- $\alpha$ antagonists and the risk of hospitalization for infection in patients with autoimmune diseases. JAMA 2011; 306: 2331-2339.

37 Kestens C, van Oijen MG, Mulder CL, et al. Adalimumab and infliximab are equally effective for Crohn's disease in patients not previously treated with anti-tumor necrosis factor- $\alpha$ agents. Clin Gastroenterol Hepatol 2013; 11: 826-831.

38 Fénix-Caballero S, Alegre-del Rey EJ, Castaño-Lara R, et al. Direct and indirect comparison of the efficacy and safety of adalimumab, etanercept, infliximab and golimumab in psoriatic arthritis. J Clin Pharm Ther 2013; 38: 286-293. 